\title{
Estimating Macroscopic Volume Delay Functions with the Traffic Density Derived from Measured Speeds and Flows
}

\author{
Rafał Kucharski and Arkadiusz Drabicki \\ Department of Transportation Systems, Cracow University of Technology, Ul. Warszawska 24, 31-155 Kraków, Poland \\ Correspondence should be addressed to Rafał Kucharski; rkucharski@pk.edu.pl
}

Received 25 July 2016; Revised 12 January 2017; Accepted 5 February 2017; Published 26 February 2017

Academic Editor: Alexandre G. De Barros

Copyright (C) 2017 Rafał Kucharski and Arkadiusz Drabicki. This is an open access article distributed under the Creative Commons Attribution License, which permits unrestricted use, distribution, and reproduction in any medium, provided the original work is properly cited.

\begin{abstract}
This paper proposes a new method to estimate the macroscopic volume delay function (VDF) from the point speed-flow measures. Contrary to typical VDF estimation methods it allows estimating speeds also for hypercritical traffic conditions, when both speeds and flow drop due to congestion (high density of traffic flow). We employ the well-known hydrodynamic relation of fundamental diagram to derive the so-called quasi-density from measured time-mean speeds and flows. This allows formulating the VDF estimation problem with a speed being monotonically decreasing function of quasi-density with a shape resembling the typical VDF like BPR. This way we can use the actually observed speeds and propose the macroscopic VDF realistically reproducing actual speeds also for hypercritical conditions. The proposed method is illustrated with half-year measurements from the induction loop system in city of Warsaw, which measured traffic flows and instantaneous speeds of over 5 million vehicles. Although the proposed method does not overcome the fundamental limitations of static macroscopic traffic models, which cannot represent dynamic traffic phenomena like queue, spillback, wave propagation, capacity drop, and so forth, we managed to improve the VDF goodness-of-fit from $R^{2}$ of $27 \%$ to $72 \%$ most importantly also for hypercritical conditions. Thanks to this traffic congestion in macroscopic traffic models can be reproduced more realistically in line with empirical observations.
\end{abstract}

\section{Introduction}

In this paper we will solve the estimation problem where traffic speed is a function of the traffic flow, generically expressed as $v=f(q)$ and further called volume delay function (VDF) or link-congestion function. The solution of the problem is a function which reproduces traffic speeds observed in field measurements. The VDF is commonly applied in static macroscopic traffic assignment to describe the resultant link travel times, as a function of flow (result of assignment) and capacity and free-flow travel time (constant parameters of the link). The purposes of this function are to reproduce congestion effects in the macroscopic model and to serve as an objective function in the assignment problem where the travel times are minimized [1]. The VDF is usually formulated in an easily integrable and differentiable form, since the assignment algorithm searches for the solution by using the integrals of VDF [2]. Unlike the physical representations of the traffic flow, the VDF allows the flow to exceed the capacity (which is by definition impossible within the traffic flow definitions). As a result, the flow volumes used in macroscopic assignment (and in turn in VDF) are not strictly related to the physically measured flows. The macroscopic flow (as we will denote it) is treated more like a demand flow which becomes delayed if it exceeds capacity. We will exploit this distinction in the proposed method.

The VDF shall reproduce both travel times and traffic flows realistically. Usually, the focus is to reproduce the actually observed flow pattern in the network, and it is well known that travel times in macroscopic model are a rough approximation neglecting fundamental traffic phenomena (such as bottlenecks, spillbacks, capacity drop, and gridlocks) which can be handled with dynamic traffic flow models [3]. The relationship between travel delay and flow volume used 
in macroscopic traffic flow models is extremely simplified and behaviorally unrealistic, yet it is commonly applied in big-scale traffic demand models [4]. Nevertheless, to improve the representativeness of resulting travel times, the VDF are usually estimated to match the observed traffic speeds/travel times, which raises following estimation issue, which we address in the paper.

In VDF the flow can become greater than capacity, while in field data measurements the vehicle flow, by definition, cannot exceed physical capacity. This raises an issue while estimating the shape of VDF. Namely, the macroscopic static traffic flow models represent the congestion with functional formulation which cannot be empirically observed and, in turn, cannot be estimated to reproduce the actual traffic speeds. Usually practitioners overcame this and estimate only the hypocritical part only for which the problem does not raise since the observed speed can be expressed with unique monotonically decreasing function of flow. The hypercritical part (when the flow starts decreasing) is usually neglected and arbitrary parameterizations are used [5].

The contribution of the paper is introducing a practical method to estimate the VDF from time-mean speed and flow overcoming issue of estimating the speeds for flows exceeding capacity. It is achieved by using the hydrodynamic relation of the fundamental diagram and more specifically by extending measured traffic flows and instantaneous speed with the proposed quasi-density. This allowed reformulating the volume delay estimation problem into the densitydelay estimation problem and thus obtaining an improved goodness-of-fit with available measurements. Finally, by expressing the macroscopic flow with a quasi-density, the estimated VDF can be used in macroscopic traffic assignment where densities are not available. This way the VDF becomes not only estimated with the empirical data, but also coherent with principal traffic flow relations.

The paper is organized as follows. The following part reviews the literature, followed by Section 2 when the method is formally introduced. It starts with introducing the estimation problem for VDF, followed with dataset description and revealing problems while estimating VDF from field data. Subsequently we introduce idea of extending measured speed and flows to point densities and reformulate the VDF estimation using them. In Section 3 we present the results of the proposed method and in Section 4 we discuss them and conclude the paper.

1.1. Literature Review. All of practically applied VDF formulations follow the basic principles of traffic flow theory; that is, the speed decreases with the increasing flow, or, equivalently, with the increasing saturation rate. Saturation rate is computed as the ratio between the flow and the capacity, with capacity being unknown and (as we show further) estimated internally within the proposed estimation problem. Reference [5] singles out mathematical and behavioral conditions which should be met by the VDF; that is, it should be described with a continuous, strictly increasing, and nonnegative function, which clearly contradicts the actual traffic flow [6]. The relation between the speed and saturation rate in VDF is highly nonlinear: initially, speed remains almost intact and rises slowly until some congestion is generated and then after reaching the assumed saturation level, speed starts to fall down significantly. The characteristic point of the VDF is when the flow equals capacity. In several VDF the formula is conditional and assumes different parameterization before and after the capacity threshold is reached. The VDF remains continuous, but the differential usually becomes much higher above this threshold, which allows separating two different regimes both in parameterization and in interpretation.

The common formulations of the volume delay functions are based on the standard BPR function (Bureau of Public Roads [7]), which has been adopted in the US guidelines and widely applied ever since in transportation planning practice [8]. A number of alternative VDF formulations have been proposed, so as to match better with observed operating conditions and road facility characteristics, which include most notably: Davidson [9], Conical [10], Akcelik [11], and Vatzek [5], as well as empirically modified BPR variations [12]. One of recurrent reasons for revisiting the standard VDF formulas in research works was identifying drawbacks in function performance for volumes approaching and exceeding the capacity rate. Studies observed that default BPR functions and their later modifications are likely either to overestimate travel times for the congested conditions [11] or lead to overestimated traffic volumes when capacity is exceeded and conversely underestimated traffic flows for relative free-flow conditions [13]. Reference [10] further noted that by adjusting the BPR parameters the travel times become more constant ("suppressed") in uncongested traffic conditions, but at the same time much more sensitive for volumes approaching and exceeding the capacity. Empirical practice shows that steepness of the speed-volume curve in congested conditions is strongly related to road-design and is different for freeways than urban arterials [11]. Reference [10] reckons that the maximal steepness rate of a VDF should be limited to limit the risk of travel time overestimation.

The VDF can be empirically observed only below the capacity rate, which divides the estimation problem into two parts: realistic curve estimation for the hypocritical part and arbitrary formulation for the hypercritical part [5]. This leads to dual approach to VDF estimation: realistic, which can be supported with empirical data in the hypocritical part; and theoretical, as it cannot be observed, in the hypercritical part. The hypercritical part of the VDF is formulated solely to be used in the assignment model, while the hypocritical part is estimated to fit the empirical observations. Other authors [2] claim that the VDF is completely unrealistic and shall be treated only as part of the traffic assignment algorithm. Importantly, [3] claims that the static assignment cannot reproduce both traffic flows and travel times realistically and further argues that the purpose of the VDF is not to reproduce the actual travel speeds, but to guarantee the convergence of the Wardropian algorithm [14] towards the user-equilibrium. Consequently, the VDF can be both easily differentiated (to obtain the search direction) and integrated. The goal function of equilibrium assignment is usually formulated with the VDF formula [15] which yields the total link travel time from the first to the last vehicle. This may however result in conflict 
between the empirical representation of the traffic flow and the performance and convergence of the traffic assignment algorithm.

Reproducing the travel time delay solely from a direct speed-volume estimation can prove to be a complex and challenging issue in practice. Empirical works, such as [16, 17], aimed to calibrate the VDF for congested traffic regimes but observed major obstacles in collecting traffic flow data for traffic states where volumes exceeded capacity-in the end, simulation models were used to generate the necessary data instead. A different and perhaps more interesting approach is to extend the scope of analysis to include the speeddensity relationship, thus exploiting a much wider extent of macroscopic fundamental diagram. Empirical estimations based on a speed-density relationship are likely to be more accurate when identifying distinct traffic conditions (freeflow, congested, and mixed traffic conditions) and the jam density limits $[18,19]$. In general overview, the idea of utilizing the speed-density relationship to estimate the speed-flow curve remains (to the best of our knowledge) relatively not much investigated in traffic modelling research. Reference [20] discusses such transition, illustrated with an example of estimating a speed-flow curve from a calibrated speeddensity one, which as a result underestimates the capacity rate; the limitation reason is the discontinuity between uncongested and congested flows (so-called capacity drop). Yet [21] compares two approaches in VDF curve estimation and argues that expressing the average speeds with volumes yields a low correlation which can be substantially improved when the speed-density relation is used instead.

\section{Method}

In this paper we argue that the VDF relation can be observed over the broader domain, by utilizing flow densities instead of flow volumes to describe resultant speeds (travel times) on network. We will demonstrate that the VDF formula can be both algorithmically efficient and provide an improved goodness-of-fit with the field data, not just for flows in the hypocritical part, but also for the hypercritical part of the fundamental diagram. To illustrate the method proposed in the paper we will use the classic BPR formula [7], which has been ever since studied in numerous research works in its either original or modified form [12] and is discussed up to now [4].

To formulate the problem, the following notation will be used:

$$
\begin{aligned}
& q: \text { flow }[\mathrm{veh}] ; \\
& v: \text { speed }[\mathrm{km} / \mathrm{h}] ; \\
& k: \text { quasi-density }[\mathrm{veh} / \mathrm{km}] ; \\
& t: \text { travel time }[\mathrm{s}] ; \\
& v_{0}: \text { free-flow speed }[\mathrm{km} / \mathrm{h}] ; \\
& q_{\max }: \text { capacity }[\mathrm{veh} / \mathrm{h}] ; \\
& k_{\max }: \text { density-at-capacity }[\mathrm{veh} / \mathrm{km}] ; \\
& a, b: \text { estimated parameters of } \mathrm{VDF}[-] ; \\
& \widehat{v}: \text { theoretical speed computed with } \mathrm{VDF}[\mathrm{km} / \mathrm{h}]
\end{aligned}
$$

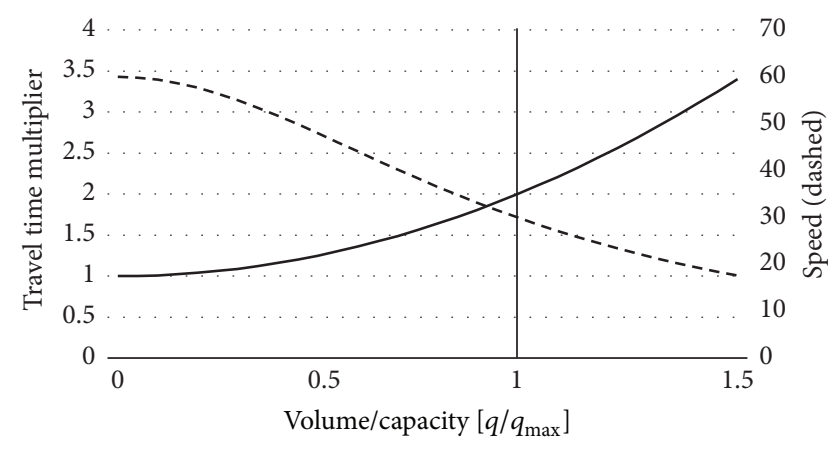

FIGURE 1: Travel time multiplier and speed in the BPR function.

$\widehat{t}$ : theoretical travel time computed with VDF [s].

To illustrate the method proposed in the paper, we will utilize the simplest BPR function, formulated with

$$
\widehat{t}=t_{0}\left(1+a \cdot\left(\frac{q}{q_{\max }}\right)^{b}\right) .
$$

It expresses travel time as a function of free-flow travel time, flow-to-capacity ratio, and the two parameters $a$ and $b$.

Figure 1 depicts travel time multiplier and speed modelled with BPR function (parameters $a=1$ and $b=2$ ), which increases steadily from one until the capacity limit and becomes even steeper afterwards. BPR (1) can be formulated relatively to express the travel time delay:

$$
\frac{\widehat{t}}{t_{0}}=\left(1+a \cdot\left(\frac{q}{q_{\max }}\right)^{b}\right)
$$

By assuming constant length the relative speed drop can be derived from BPR function (1) as inverse of travel times:

$$
\widehat{v}=\frac{v_{0}}{\left(1+a \cdot\left(q / q_{\max }\right)^{b}\right)} .
$$

In the paper, we discuss the estimation problem of the BPR function where we look for optimal values of parameters $a$ and $b$. The objective is to reproduce the observed data with BPR function; that is, the modelled speeds should match the measured ones, which can be formalized with the following least-squares formula computed as the sum of squared difference between modelled and observed speeds for all the observations $\tau$ in the dataset $T$ :

$$
\underset{a, b}{\arg \min }\left(\sum_{\tau \in T}\left|\widehat{v}_{\tau}-v_{\tau}\right|^{2}\right)
$$

We do not analyze the solution of problem (4) itself, since it is straightforward and can be solved with any linear solver (we formulated the problem for the least-squares method). Instead, we will focus on how to support the estimated BPR function with the measured data, which is nontrivial, as discussed. When using the relative formulation of VDF, the capacity and free-flow-speed rates need to be known a priori, whereas if using its absolute formulation, 
both capacity and free-flow speed can become the estimated (unknown) parameters themselves. This way, problem (4) can be extended to (5) in which the physical parameters (freeflow speed and capacity) are estimated along with the BPR parameters, which is way more practical.

$$
\underset{a, b, t_{0}, q_{\max }}{\arg \min }\left(\sum_{\tau \in T}\left|\widehat{v}_{\tau}-v_{\tau}\right|^{2}\right) .
$$

2.1. Speed-Flow Field Measurements. We illustrate the proposed method with field measurement data from the Warsaw count locations where the vehicle flows and speeds are measured. Vehicle flow is continuously measured in over hundred locations in crucial points across the Warsaw road network, some of which are equipped with the double induction loops, capturing also instantaneous vehicle speeds [22]. Although this provides huge amount of data which can be used to solve the proposed VDF estimation problem, it has some significant limits when applying to fundamental traffic flow relations, as discussed in Section 2.3.

We used measurements collected from one loop placed at the main road site at the boundary of Warsaw. It measures traffic flows towards the city centre on the three-lane arterial road with a posted speed limit of $70 \mathrm{~km} / \mathrm{h}$ which becomes highly congested during peak hours. For all the approaching vehicles, instantaneous speeds were measured and the datasets were aggregated over 15-minute time intervals. We analyzed data collected for six months was used, which covered 15 thousand records and almost 6 million vehicles (for further references see [23]). It should be noted that the entire 6-month dataset can be used for the estimation purposes and does not have to be restricted to the working days and/or peak hours only, since the estimated relation is a physical phenomenon and depends mostly on capacity and resultant speed drops each time congestion arises. What is more, road sections which are similar to each other (i.e., those with the same road class, speed limit, intersection design, lane width and number of lanes, pavement quality, etc.) can be handled jointly so as to enlarge the input data samples. It is worth noting that to estimate the VDF the data can be used only if the congestion is observed and measured. For the count locations which do not become congested at any time of a day, the VDF cannot be estimated, as it is not possible to analyze the resultant speed drops observable during congestion, regardless of the big number of observations. The analysis of our field data measurements did not reveal any significant errors, which implies that these can be therefore assumed to be consistent; nonetheless we excluded some outlier values. The outliers observed during the hypocritical (uncongested) traffic states were assumed to be the measurement errors. On the other hand, since the randomness of traffic conditions in the hypercritical (congested) conditions is high, we studied the outliers of the congested state carefully, to exclude the actual measurement errors and preserve the representative measurements of the congested traffic.

Figure 2 presents the aerial view of the measurement site. Instantaneous traffic speeds and flow volumes are measured

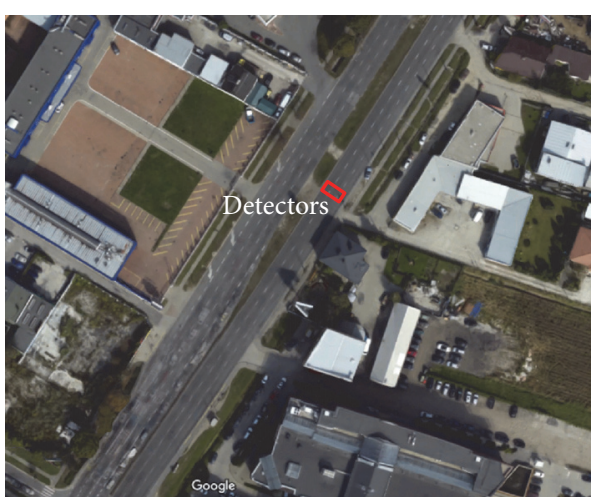

Figure 2: Measurement site, Al. Krakowska, Warsaw, Poland, (c) Google Satellite.

by means of the stationary induction loops, separately for each lane. The input data is aggregated to 15 -minute time intervals and $10 \mathrm{~km} / \mathrm{h}$ speed intervals. Values below $10 \mathrm{~km} / \mathrm{h}$ are neglected, as well as the last interval which contains all the vehicles passing the site with speeds exceeding $100 \mathrm{~km} / \mathrm{h}$. Single database record in the database is the number of detected vehicles in a given 15-minute interval in the individual $10 \mathrm{~km} / \mathrm{h}$ speed range. From such data we obtain following fundamental characteristics:

(i) Average weighted instantaneous speed, given with

$$
v_{\tau}=\frac{\sum_{n}\left(q_{\tau, n} \cdot \bar{v}_{n}\right)}{\sum_{n} q_{\tau, n}}
$$

where $q_{\tau, n}$ is the number of vehicles passing the detector during 15-minute interval $\tau$ with a speed within range $n$ and $\bar{v}_{n}$ is the middle speed value of the range.

(ii) Capacity, initially assumed to be the 95th percentile of the measured vehicle flows and further estimated within VDF estimation.

(iii) Free-flow speed initially assumed to be the 85th percentile of the measured speed and further estimated within VDF estimation.

(iv) Hourly vehicle flow: for 15-minute flow aggregation, we estimate the hourly flow rate by multiplying the 15-minute flow rate by four; this enables us to trace short-term flow variations, which could otherwise disappear if, for example, the moving sum is used.

(v) Dummy instantaneous travel time multiplier, computed from the speed drop rate with $t / t_{0}=v_{0} / v_{\tau}$. Figure 3 and Table 1 present the variability of the measured traffic flow rates and travel times over a total six-month measurement period.

Data shown in Figure 3 and Table 1 will be used in the estimation through the observed flow $q$ in a given 15-minute interval and mean speed computed with (6). The observed speed derived from observations with (6) is compared in the estimation problem (4) with the theoretical speed computed 

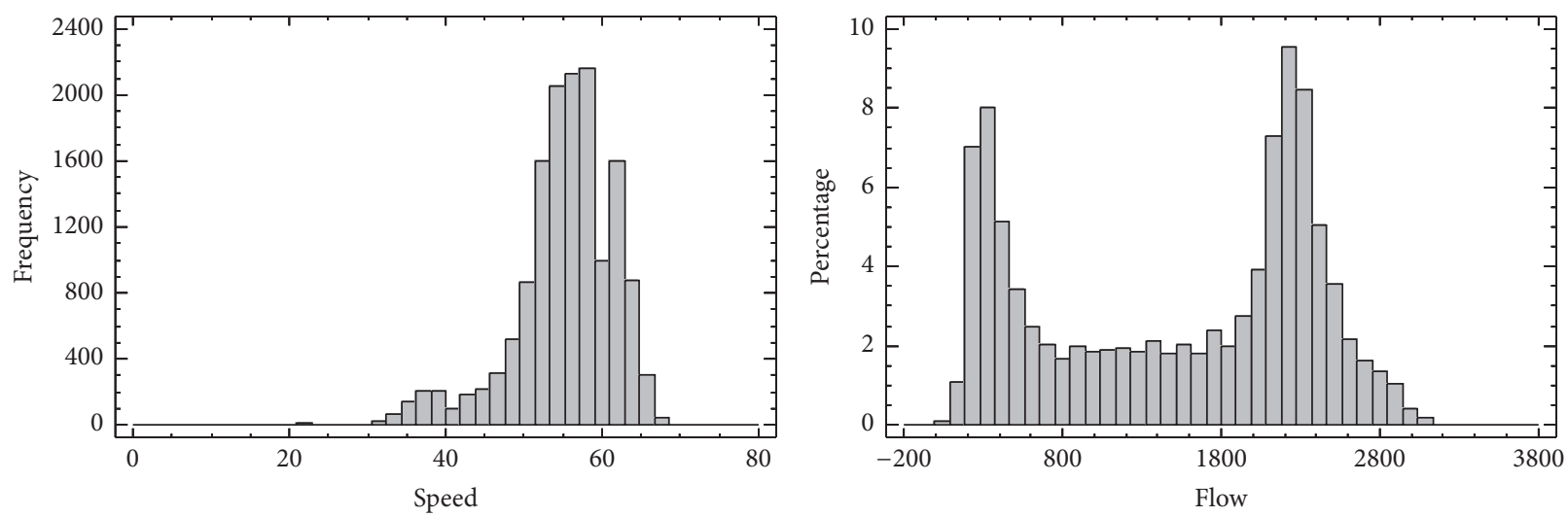

FIgURe 3: Traffic speed and flow histograms.

TABLE 1: Flow and speed percentiles.

\begin{tabular}{lcc}
\hline Percentile & Speed & Flow \\
\hline 80 & 60 & 2316 \\
85 & 61 & 2376 \\
90 & 62 & 2476 \\
95 & 63 & 2640 \\
99 & 66 & 2900 \\
99,99 & 70 & 3128 \\
\hline
\end{tabular}

with the BPR (3) for a given flow observed in respective 15-minute interval. Mind that better speed approximation could be obtained with the harmonic mean [6] rather than arithmetic mean which can overestimate the actual spacemean speed.

\subsection{Problem with Estimating VDF from the Field Measure-} ments. The directly observed measures allow us to plot the correlation between observed instantaneous speeds against the traffic flows $\widehat{v}=f(q)$, which is the relationship supposed to be represented with the VDF like BPR (3). Yet, the graph (Figure 4) clearly shows that not only are the plot shapes different, but there is no unique functional relation between speed and flow. Measured vehicle flow starts decreasing after the capacity rate is reached and, as a result, for a given flow the two different speed ranges are commonly observed: hypoand hypercritical. For instance, the speed rate for the flow of $1800 \mathrm{veh} / \mathrm{h}$ can be equal to around $55 \mathrm{~km} / \mathrm{h}$ for hypocritical conditions and to around $35 \mathrm{~km} / \mathrm{h}$ for hypercritical conditions. This contradicts the fundamental assumptions of the VDF, namely, that the travel time multiplier is a continuous, monotonic, strictly increasing function of the flow [5]. Using such data to estimate the function (1) would lead to somewhat incoherent conclusions. Figure 5 depicts the travel time multiplier (computed from the speed drop equation $\hat{t} / t_{0}=v_{0} / \widehat{v}$ ) as a function of flow. Estimation of the parameters of function (2) with correlation observed in Figure 5 is impossible and results in a very poor goodness-offit and/or erroneous approximation. The red line in Figure 5

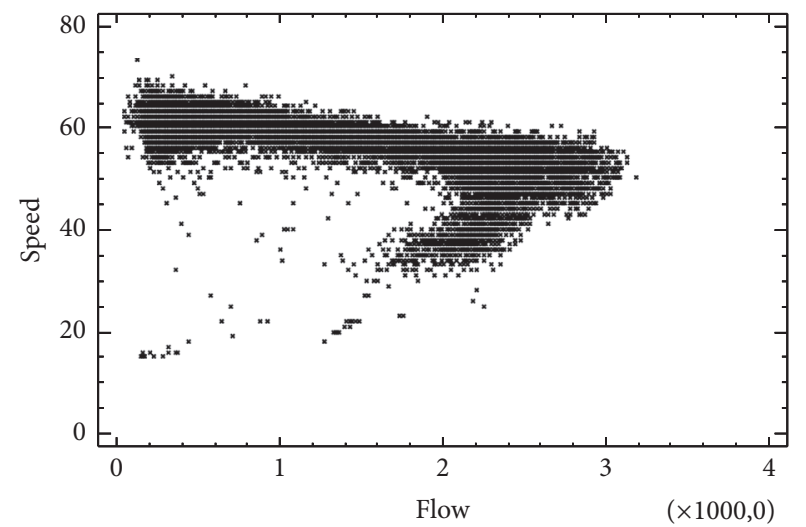

FIGURE 4: Average instantaneous speed [ $\mathrm{km} / \mathrm{h}$ ] against hourly flow [veh/h].

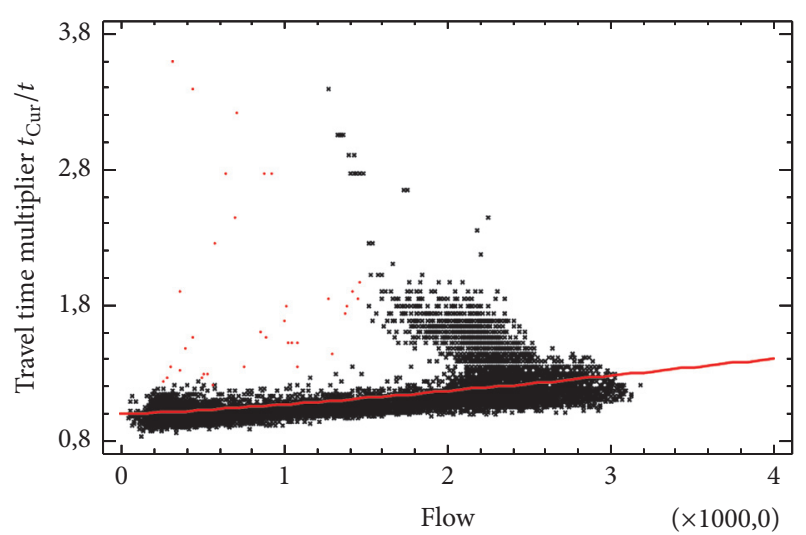

FIGURE 5: Travel time multiplier [-] against hourly flow [veh/h] and the BPR function estimated for this data (red) with output parameters equal to $a \approx 1$ and $b=1.27$.

shows the results of the BPR estimation directly from the speed and flow; to evaluate the goodness-of-fit we use $R^{2}$ expressed with the following, where $\tau$ is a single 15 -minute 
observation in the dataset $T$ and $\bar{v}$ is the mean speed in the whole dataset.

$$
R^{2}=1-\frac{\sum_{\tau \in T}\left(v_{\tau}-\bar{v}\right)^{2}}{\sum_{\tau \in T}\left(\widehat{v}_{\tau}-\bar{v}\right)^{2}} .
$$

Estimating function fitted the data of Figure 5 with $R^{2}$ of only $27 \%$ and yielded the BPR parameters $a \approx 1$ and $b=$ 1.27. What is more, such estimation underestimates speeds for hypocritical part, since the low speeds of hypercritical bias it. Further implementation of such BPR function in a macroscopic model will most likely result in flat travel times, even for flows strongly exceeding capacity, and thus lead to significant errors in the assignment results. This demonstrates that there is no empirical evidence of the flowspeed relation postulated in VDF (Figure 1). Based on the above, the proper shape of VDF cannot be directly observed in the empirical data, and consequently it cannot be estimated in a straightforward manner. In the following section we present an alternative solution approach to this issue.

2.3. Extending the Measured Speed and Flow with QuasiDensity. To overcome the problem stated above, let us utilize the fundamental hydrodynamic relation between speed and flow and introduce the third measure of the fundamental diagram: density, denoted by $k$ [24]. The fundamental relation between those three variables is described with (8), which states that the vehicle flow during a given time period equals the flow density multiplied by the flow speed [25]:

$$
q=k v \text {. }
$$

Mind that the above holds true only under certain conditions (stationary homogeneous traffic [26]) and, importantly, not for instantaneous speed but for space-mean speed. Density (understood as number of vehicles on the road section divided by its length) can be directly obtained only if the whole length of road section is observed, which is in practice hard to achieve. In the majority of practical cases, only stationary measurements are available, and the density can only need to be approximated from instantaneous speed and flow values. There are numerous ways of deriving it; for broader discussion of this problem we refer to $[6,26]$. Unfortunately, more exact formulas require either spacemean speed or occupancy rate. Since neither of them was available in field data results, we can only approximate density from the classic hydrodynamic equation (8).

Thus, being aware of the limitations and possible biases, for the purposes of the proposed method, we will derive density from measured time-mean speed and time-mean flow with (9) and further denote it as quasi-density $k$ :

$$
k=\frac{q}{v} .
$$

This can be deemed appropriate and sufficient for the method proposed in the paper, since it is used only as unobserved intermediate variable and the actual density (i.e., number of vehicles per kilometer) - as it will be seen in further sections-is not required. Quasi-density computed $(\times 1000,0)$

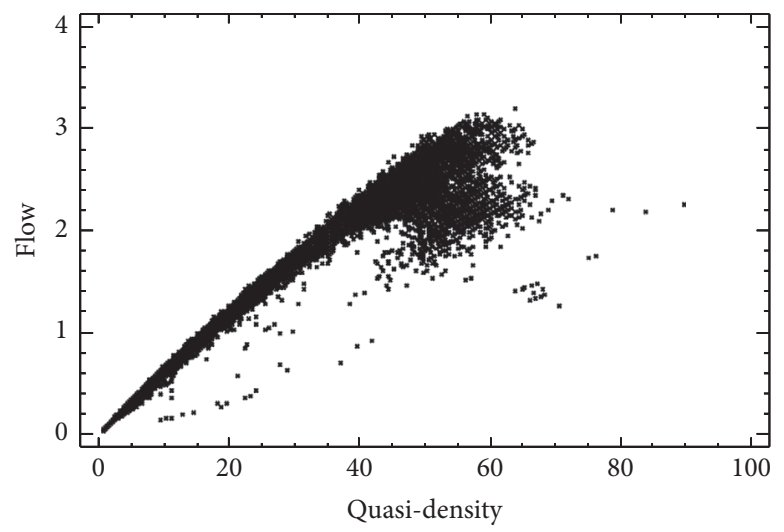

FIGURE 6: Fundamental diagram; hourly flow (veh/h) against quasidensity (veh/km).

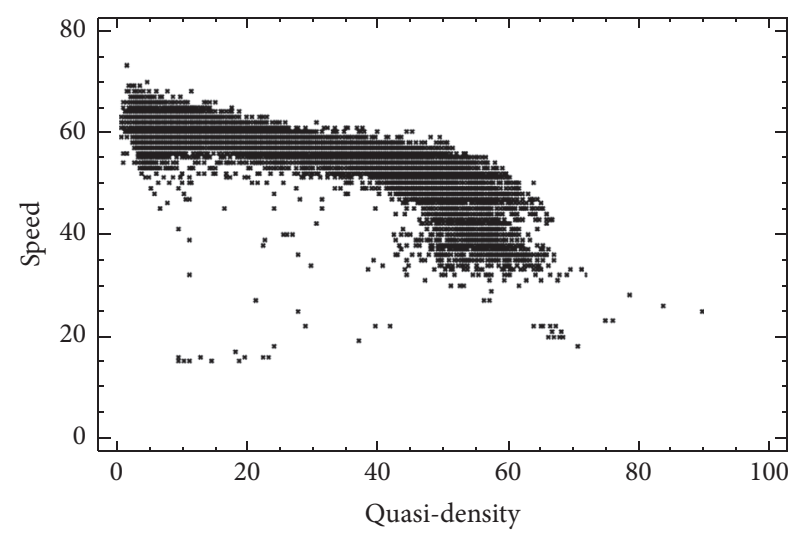

FIGURE 7: Speed $(\mathrm{km} / \mathrm{h})$ against quasi-density $(\mathrm{veh} / \mathrm{km})$.

with (9) will be used in an abstract way, not in a strict physical sense. The purpose of such derivation is to obtain a more explicit and adequate variable to reformulate the VDF (2).

The fundamental diagram depicting traffic flow against quasi-density $q=f(k)$ for the observed field data is shown in Figure 6. It resembles the common fundamental diagram shapes reported in the literature [27-29], which proves that the fundamental traffic flow relations are preserved also when using quasi-density.

Figure 7 shows the speed as a function of quasi-density $v=f(k)$, and Figure 8 shows the travel time multiplier as a function of quasi-density $t_{\text {cur }} / t=f(k)$. Interestingly, both resemble now the typical BPR function (3) where speed decreases steadily until capacity threshold is reached and starts to fall sharply when capacity is exceeded (compare the analytical BPR plots in Figure 1 with empirical relations shown in Figures 7 and 8). This was not possible to attain for the empirical flow-speed relationship, which not only had a different shape but did not have unique functional form at all. While the flow-speed relation (Figure 4) does not have a unique functional form, the density-speed relation (Figure 7) does have one; that is, for a given density rate we can estimate 


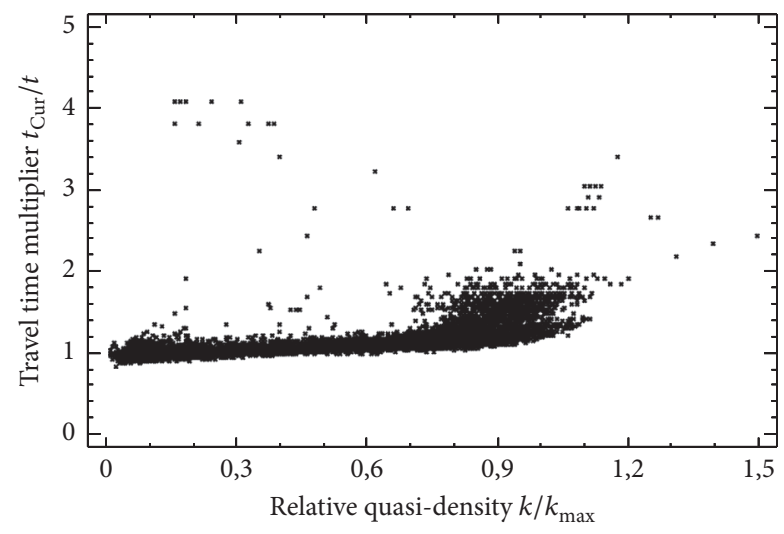

FIGURE 8: Travel time multiplier against quasi-density (veh/km).

a corresponding unique flow-speed value (Figure 7). We will utilize this finding to reformulate the BPR functions to make them more realistic.

2.4. Method to Estimate Macroscopic VDF with Quasi-Density. Once we stated that the BPR function shape is observed against density, not a flow, we will propose the way to obtain densities in the macroscopic assignment where they are not available. To this end, let us first further exploit distinction between the measured (physical) flow $q$ and the flow of macroscopic model (further denoted by $q_{\mathrm{VDF}}$ ) used in the VDF. As already signalized in introduction, both of them are different and have different physical interpretations [5]. Measured flow $q$ is strictly constrained by the traffic flow dynamics and any observation contradicting them might only result from measurement errors. Consequently, the measured flow cannot exceed capacity and drops down when the demand volume exceeds the maximal density (Figure 6). On the other hand, the macroscopic flow is constrained only by the assumptions of the macroscopic traffic flow model, which is by itself a significant simplification. The macroscopic flow may exceed the capacity, and it gets severely delayed but in general is allowed. The macroscopic flow is in principle proportional to the demand (i.e., the higher the demand is, the greater the macroscopic flow is), while in reality the actual flow pattern is essentially different (i.e., it grows with the demand only until capacity limit is reached and falls down afterwards).

We postulate to consider macroscopic flow in terms of the demand flow and state that the best approximation possible of macroscopic flow can be achieved in relation to the density, not the flow. Similarly to the macroscopic flow, density grows with the increasing demand volume but reaches its maximum value way above the capacity rate, at the "traffic-jam" density conditions. If so, let us assume that the macroscopic flow shall be understood in terms of density rather than the measured flow and reformulate the traditional VDF flow-speed relation into the density-speed relation.

Since density is not available in the macroscopic static models it cannot be used directly in the VDF formulas. To overcome this problem, let us propose the following mapping from observed quasi-densities to flows of the macroscopic assignment. Such linear mapping is defined with (10), where the VDF flow equals capacity multiplied by the density to density-at-capacity ratio.

$$
q_{\mathrm{VDF}} \doteq q_{\max } \cdot \frac{k}{k\left(q_{\max }\right)} .
$$

Density-at-capacity $k\left(q_{\max }\right)$ is the density at which actual flow $q$ reaches maximum and can be read from the fundamental diagram (Figure 6). The inverse of this mapping is given with (11) where density is expressed as the function of constant values read from the empirically observed fundamental diagram (capacity and density-at-capacity) and macroscopic flow $\left(q_{\mathrm{VDF}}\right)$. Thus, by means of (11), the quasidensity value can be evaluated from the macroscopic flow.

$$
k \doteq q_{\mathrm{VDF}} \cdot \frac{k\left(q_{\max }\right)}{q_{\max }} .
$$

We stated that the BPR function can be observed empirically, yet not as a function of flow, but as a function of quasidensity $v=f(k)$. Thus, let us redefine the BPR formula (1) and substitute the flow with (10) to obtain (12).

$$
\widehat{t}=t_{0}\left(1+a \cdot\left(\frac{k}{k\left(q_{\max }\right)}\right)^{b}\right) .
$$

Quasi-density is used here as a substitute of the flow, while the density-at-capacity $k\left(q_{\max }\right)$ substitutes the capacity. The observed shape of density-speed relation (Figure 7) is similar to the BPR shape (Figure 1), so we presume that the estimation process will result in a high goodness-of-fit ratio. This way we obtained the following two formulations of the BPR function. The first one is used for further estimation and is formulated as follows:

$$
\begin{aligned}
\widehat{v} & =\frac{v_{0}}{\left(1+a \cdot\left(k / k\left(q_{\max }\right)\right)^{b}\right)} \\
& =\frac{v_{0}}{\left(1+a \cdot\left((q / v) / k\left(q_{\max }\right)\right)^{b}\right)} .
\end{aligned}
$$

In this formulation, modelled speed is directly expressed with the observed traffic flow and speed values along with estimated parameters $v_{0}$ and $q_{\max }$. We obtain a straightforward estimation and the shape of model function is directly observable when plotted against the field data. Moreover, (13) yields straightforward estimation problem, since we match the measured speeds also with the modelled speeds and not only with measured flows.

Once the BPR parameters have been estimated, we need to reformulate the BPR function for the purposes of macroscopic assignment algorithm. To this end, we use the inverse mapping from densities to macroscopic flow (11) and substitute the quasi-density in formula (13); all the unknown 
parameters cancel out to the final formula (14), which is principally the initial BPR formula given by (3):

$$
\begin{aligned}
\widehat{v} & =\frac{v_{0}}{\left(1+a \cdot\left(k / k\left(q_{\max }\right)\right)^{b}\right)} \\
& =\frac{v_{0}}{\left(1+a \cdot\left(q_{\mathrm{VDF}} \cdot\left(k\left(q_{\max }\right) / q_{\max }\right) / k\left(q_{\max }\right)\right)^{b}\right)} \\
& =\frac{v_{0}}{\left(1+a \cdot\left(q_{\mathrm{VDF}} / q_{\max }\right)^{b}\right)} .
\end{aligned}
$$

The output BPR function formulated with (14) can be applied in macroscopic assignment. The two parameters $a$ and $b$ are constant and are estimated with (13) along with free-flow speed $v_{0}$ and capacity $q_{\max }$. The only variable is the macroscopic flow $q_{\mathrm{VDF}}$, which is available in the macroscopic traffic assignment model. This way, we are able to estimate the BPR-wise function and apply it in the macroscopic model as illustrated in subsequent section.

\section{Results of Estimating VDF with Quasi-Density}

Let us apply the above reformulations and propose the final form of the estimation problems (4) and (5), which can be solved with the empirical measurement data. As advocated above, let us express the travel time multiplier as a function of quasi-density (12). The quasi-density $k$ used in this formulation is computed from the measured time-mean speed and flow with (9) and the density-at-capacity $k\left(q_{\max }\right)$ is equivalent to the peak value read from the fundamental diagram (Figure 6). In this particular case, the free-flow speed identified as the 85 th percentile was equal to $61 \mathrm{~km} / \mathrm{h}$, the capacity identified as the 95th percentile of 15-minute flow was equal to $2640 \mathrm{veh} / \mathrm{h}$, and $k\left(q_{\max }\right)$ was estimated to be ca. $60 \mathrm{veh} / \mathrm{km}$, which is ca. $16 \mathrm{~m} / \mathrm{veh}$. By applying these values we can solve the estimation problem (4), utilizing (12) as the travel time function. Results of the estimation are shown in Figure 9. BPR parameters are estimated to be equal to $a=$ 0.52 and $b=3.47$, and the estimation process yielded $R^{2}$ of $65 \%$ for the total sample of 14,666 measurements (around 50 measurement records were excluded due to identified measurement errors). The resulting shape is promising and matches the theoretical expectations (discussed earlier). The BPR function with such parameterization implies high delays when capacity is exceeded: the resultant travel time multiplier is around 3.0 for the demand volume equal to $150 \%$ of capacity rate. Presumably, more complex VDF formulations [5] could result in better goodness-of-fit, while for illustrative purposes we used basic BPR formulation.

Finally, let us further relax problem (4) and estimate both speed and capacity (density-at-capacity in this formulation) with the BPR function as formulated in (5). The estimation of such formulated problem is also possible; and output results are similar. Since the free-flow speed becomes a parameter now, it is convenient to express the BPR function with the codomain being speed rather than delay; the plotted

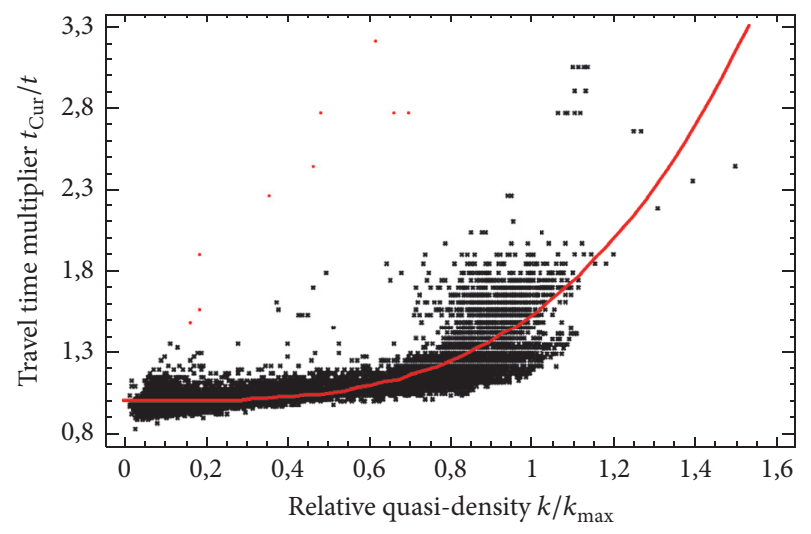

FIGURE 9: BPR function (red) fitted to $t_{\text {Cur }} / t$ against $k / k_{\max }$ data, $a=$ 0.52 and $b=3.47$.

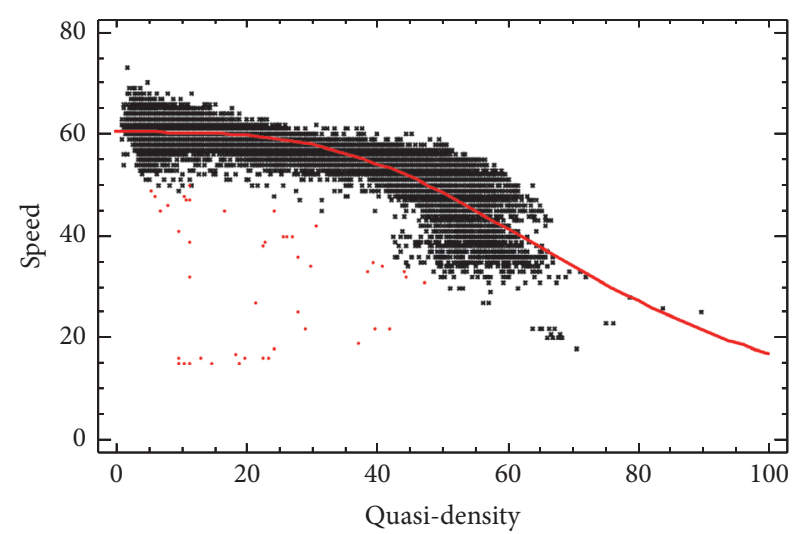

FIGURE 10: Speed versus density with the estimated BPR function, $v_{0}$ and $k_{\max }(\mathrm{red})$, solution of the extended estimation problem (5).

function shape is now different due to using (3) instead of (1). By solving such estimation problem, the free-flow speed is estimated at $60.3 \mathrm{~km} / \mathrm{h}, k_{\max }$ is estimated at $61.5 \mathrm{veh} / \mathrm{km}$, and the BPR parameters are estimated at $a=0.49, b=3.41$, respectively. Importantly, the estimated model fitted field data values with $R^{2}$ of $72 \%$, a significant improvement compared to the baseline VDF results. The plot of observed speed versus quasi-density $k$ is depicted with a red line in Figure 10.

\section{Summary and Conclusions}

The classical VDF assumes that the flow can exceed capacity, which is somehow acceptable for the macroscopic assignment problem, yet it is hardly consistent with actual field measurements (since, by definition, the capacity is the maximum flow which can be possibly observed). Consequently, the VDF cannot be estimated directly from the measured flows (at least for the hypercritical part: see Figure 4). We propose the method to overcome this issue by employing the fundamental hydrodynamic relations between traffic flow variables $q=$ $k v$, which underlies most of the contemporary higher-level traffic flow models. Our proposal introduced in this paper is to estimate the VDF by using density instead of flow. 
Thanks to such interpretation, a BPR-like plot shape of the delay-density function (Figure 9) was obtained from the observed data. With such formulation one can consistently use both the hypo- and hypercritical part of the fundamental diagram to estimate the speed-flow function. By utilizing the proposed method, travel times computed in the macroscopic assignment are likely to be more realistic. The method is illustrated with an example where BPR parameters have been consistently estimated with the density derived from measured flows and instantaneous speeds. The goodnessof-fit was improved from $R^{2}$ of $27 \%$ to $72 \%$, which implies that output results were much more coherent with the field data. The proposed method cannot substitute the realistic traffic flow models, which are more accurate and superior in reproducing the traffic phenomena; nevertheless it is adequate for macroscopic models which are broadly used in the field of transportation modelling. The proposed method does not change the functional form of the VDF, which is still convex and monotonically increasing function of macroscopic flow and can still be used in assignment algorithms providing efficient objective function converging to Wardrop equilibrium.

This research can be further extended to cover another data sources like floating car data or space-mean densities. Also the exposed speed-density relations can advocate new mathematical formulations of VDF which will yield better fit. The naivve least-squares formulation of the estimation problem might be revised to weight more the hypercritical observations (which are crucial to reproduce congestion). Finally the proposed method shall be applied on the real-size strategic model to show its applicability.

\section{Competing Interests}

The authors declare that there is no conflict of interests regarding the publication of this paper.

\section{References}

[1] M. Fukushima, "A modified Frank-Wolfe algorithm for solving the traffic assignment problem," Transportation Research. Part B. Methodological. An International Journal, vol. 18, no. 2, pp. 169-177, 1984.

[2] G. Gentile and K. Noekel, "Linear user cost equilibrium: the new algorithm for traffic assignment in VISUM," in Proceedings of the European Transport Conference, Noordwijkerhout, The Netherlands, October 2009.

[3] G. Gentile, L. Meschini, and N. Papola, "Macroscopic arc performance models with capacity constraints for within-day dynamic traffic assignment," Transportation Research Part B: Methodological, vol. 39, no. 4, pp. 319-338, 2005.

[4] W. Wong and S. C. Wong, "Network topological effects on the macroscopic Bureau of Public Roads function," Transportmetrica A: Transport Science, vol. 12, no. 3, pp. 272-296, 2016.

[5] W. Jastrzebski, "Volume delay functions," in Proceedings of the 15th International EMME/2 Users Group Conference, Vancouver, Canada, October 2000.

[6] M. Treiber and A. Kesting, Traffic Flow Dynamics: Data, Models and Simulation, Springer, Berlin, Germany, 2013.
[7] Bureau of Public Roads, Traffic Assignment Manual, Department of Commerce, Urban Planning Division, Washington, DC, USA, 1964.

[8] D. Branston, "Link capacity functions: a review," Transportation Research, vol. 10, no. 4, pp. 223-236, 1976.

[9] K. B. Davidson, "A flow travel time relationship for use in transportation planning," in Proceedings of the 3rd Australian Road Research Board (ARRB '66) Conference, vol. 3, no. 1, Sydney, Australia, 1966.

[10] H. Spiess, "Technical note-conical volume-delay functions," Transportation Science, vol. 24, no. 2, pp. 153-158, 1990.

[11] R. Akcelik, "Travel time functions for transport planning purposes: Davidson's function, its time dependent form and alternative travel time function," Australian Road Research, vol. 21, no. 3, 1991.

[12] R. Moses, E. Mtoi, S. Ruegg, and H. McBean, "Development of speed models for improving travel forecasting and highway performance evaluation," Final Report, Florida Department of Transportation (FDOT), 2013.

[13] R. Singh and R. Dowling, "Improved speed-flow relationships: application to transportation planning models," in Proceedings of the 7th TRB Conference on the Application of Transportation Planning Methods, Boston, Mass, USA, 2002.

[14] J. G. Wardrop, "Some theoretical aspects of road traffic research," 1952.

[15] M. Frank and P. Wolfe, "An algorithm for quadratic programming," Naval Research Logistics Quarterly, vol. 3, no. 1-2, pp. 95110, 1956.

[16] R. Dowling and A. Skabardonis, "Improving average travel speeds estimated by planning models," Transportation Research Record, vol. 1366, pp. 68-74, 1993.

[17] A. Skabardonis and R. Dowling, "Improved speed-flow relationships for planning applications," Transportation Research Record, no. 1572, pp. 18-23, 1997.

[18] B. Coifman, "Empirical flow-density and speed-spacing relationships: evidence of vehicle length dependency," Transportation Research Part B: Methodological, vol. 78, pp. 54-65, 2015.

[19] S. Omar and C. Mallikarjuna, "Analysis of the macroscopic relations for no-lane based heterogeneous traffic stream," Procedia Engineering, vol. 142, pp. 243-250, 2016.

[20] N. C. Duncan, "A further look at speed/flow/concentration," Traffic Engineering \& Control, vol. 20, no. 10, pp. 482-483, 1979.

[21] P. Olszewski, H. S. L. Fan, and Y.-W. Tan, "Area-wide traffic speed-flow model for the Singapore CBD," Transportation Research Part A: Policy and Practice, vol. 29, no. 4, pp. 273-281, 1995.

[22] Y.-K. Ki and D.-K. Baik, "Model for accurate speed measurement using double-loop detectors," IEEE Transactions on Vehicular Technology, vol. 55, no. 4, pp. 1094-1101, 2006.

[23] R. Kucharski and G. Gentile, "Indirect observation of rerouting phenomena in traffic networks-case study of warsaw bridges," Archives of Transport, vol. 32, no. 4, pp. 29-41, 2014.

[24] C. F. Daganzo, "A behavioral theory of multi-lane traffic flow. Part I: long homogeneous freeway sections," Transportation Research Part B: Methodological, vol. 36, no. 2, pp. 131-158, 2002.

[25] C. F. Daganzo and N. Geroliminis, "An analytical approximation for the macroscopic fundamental diagram of urban traffic," Transportation Research Part B: Methodological, vol. 42, no. 9, pp. 771-781, 2008.

[26] N. H. Gartner, C. J. Messer, and A. K. Rathi, Eds., Monograph on Traffic Flow Theory, Federal Highway Administration, Washington, DC, USA, 1997. 
[27] D. Helbing, "Derivation of a fundamental diagram for urban traffic flow," European Physical Journal B, vol. 70, no. 2, pp. 229241, 2009.

[28] M. Treiber, A. Kesting, and D. Helbing, "Three-phase traffic theory and two-phase models with a fundamental diagram in the light of empirical stylized facts," Transportation Research Part B: Methodological, vol. 44, no. 8-9, pp. 983-1000, 2010.

[29] M. Rickert, K. Nagel, M. Schreckenberg, and A. Latour, "Two lane traffic simulations using cellular automata," Physica A: Statistical Mechanics and its Applications, vol. 231, no. 4, pp. 534550, 1996. 


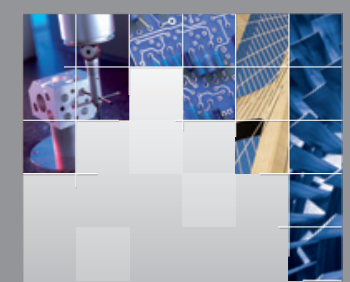

\section{Enfincering}
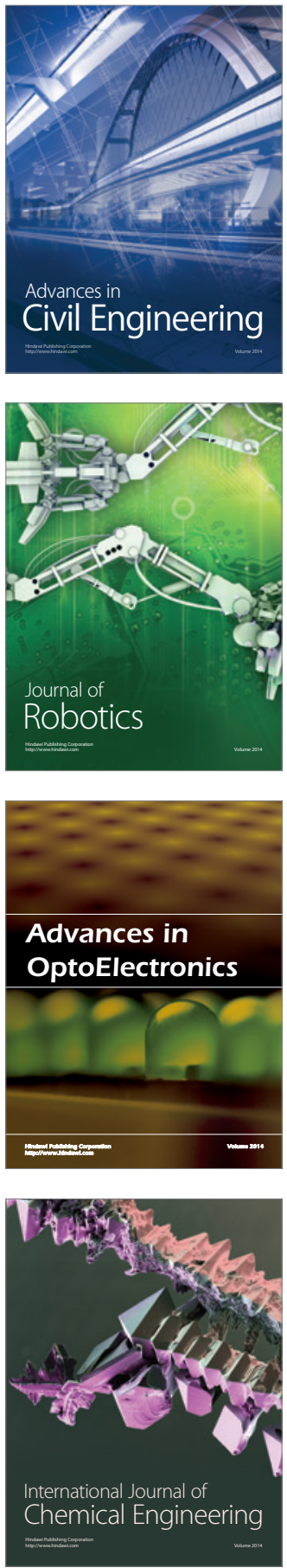

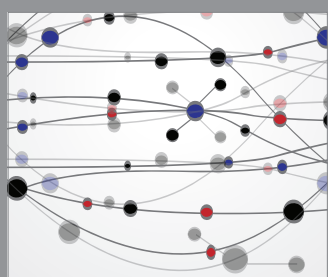

The Scientific World Journal

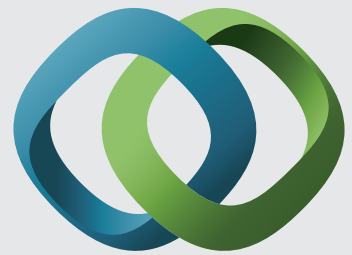

\section{Hindawi}

Submit your manuscripts at

https://www.hindawi.com
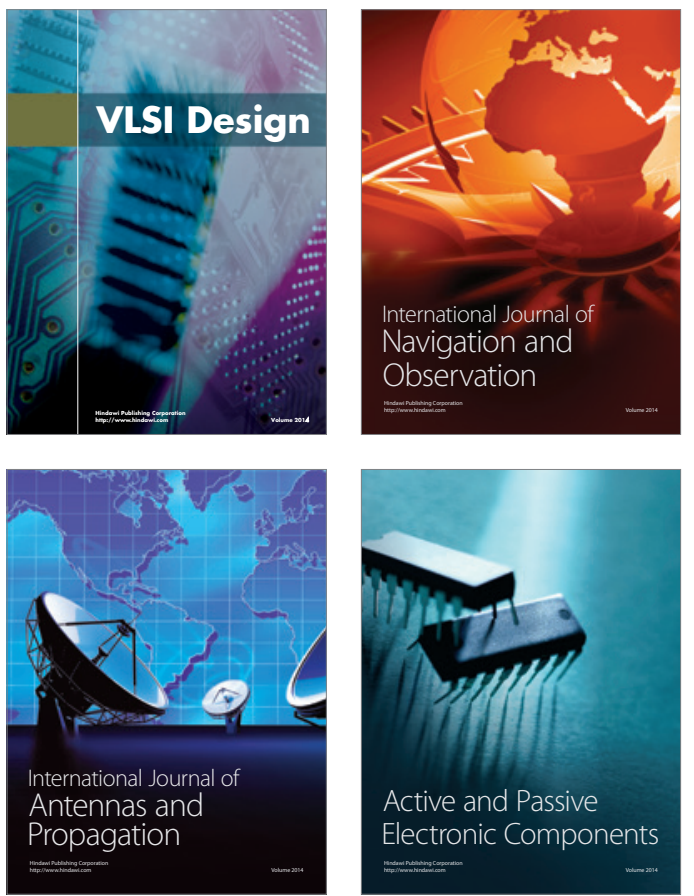
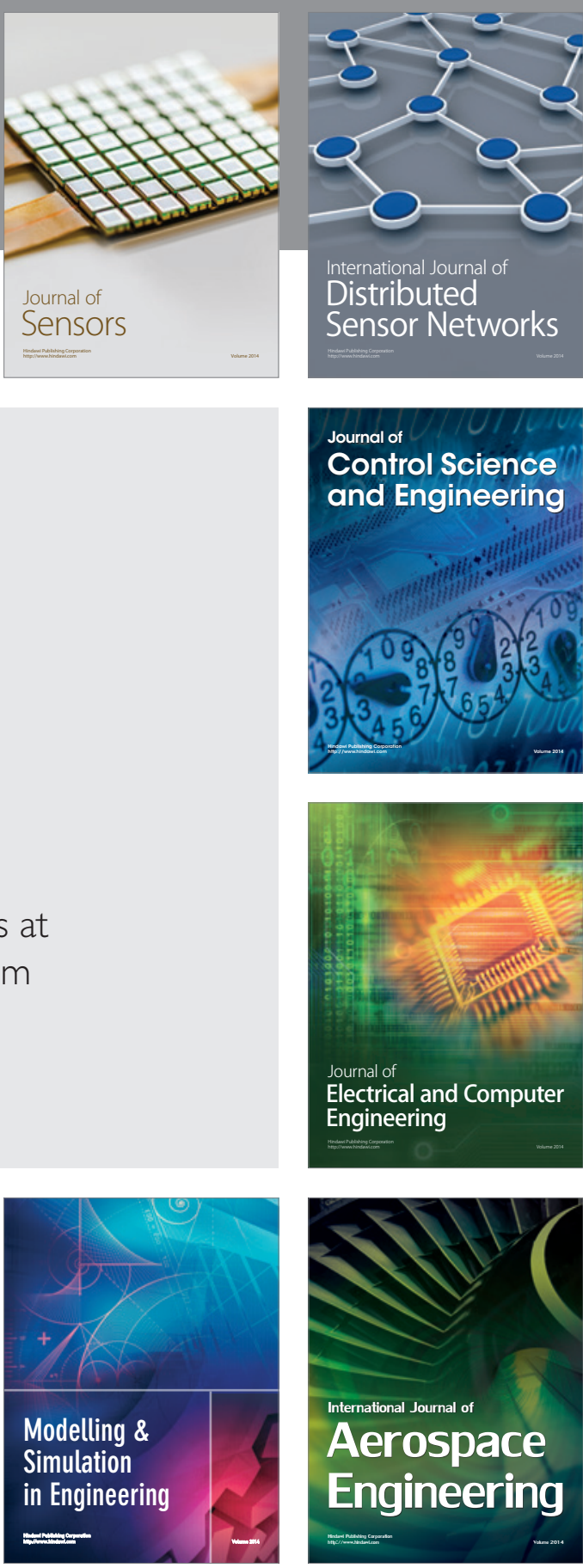

International Journal of

Distributed

Sensor Networks

$-$

Joumal of

Control Science

and Engineering
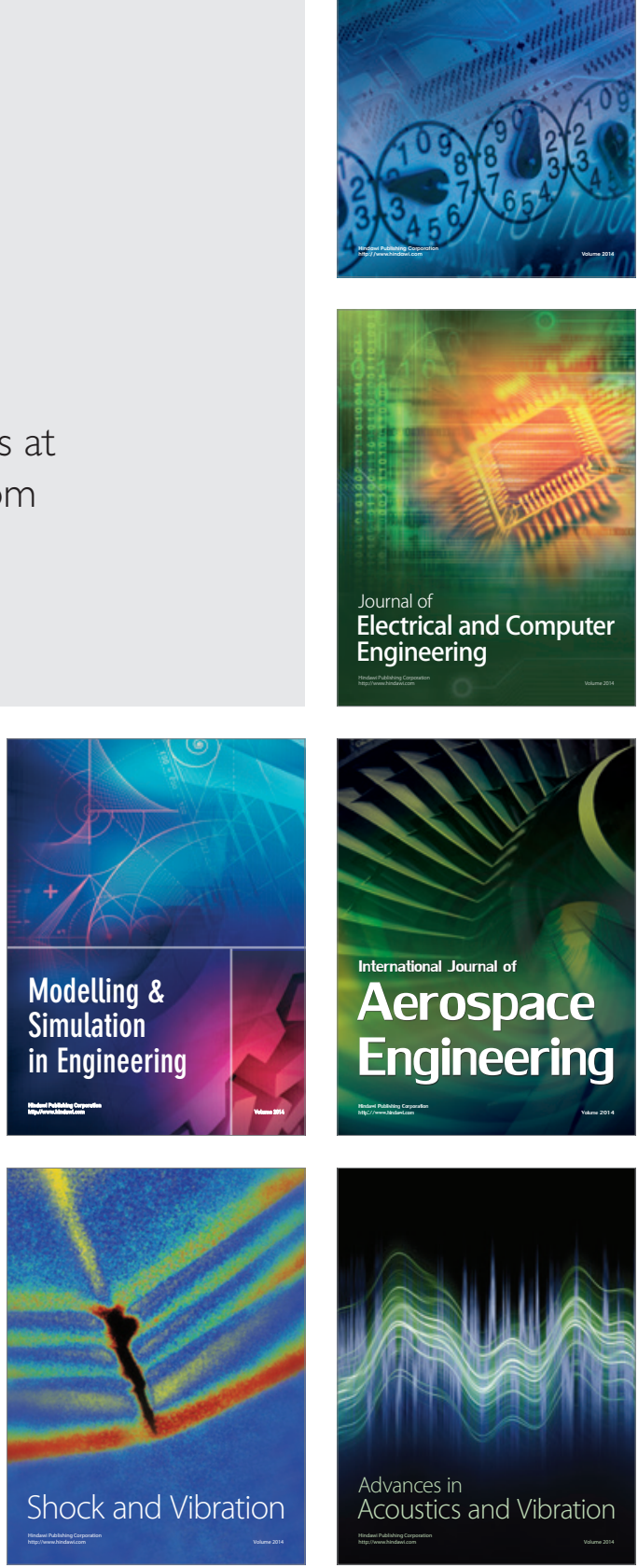